\title{
COMPARISON BETWEEN ANALYTIC CAPACITY AND THE BUFFON NEEDLE PROBABILITY
}

\author{
TAKAFUMI MURAI
}

\begin{abstract}
We show that analytic capacity and the Buffon needle probability are not comparable.
\end{abstract}

1. Introduction. For a compact set $E$ in the complex plane $\mathbf{C}$, we denote by $H^{\infty}\left(E^{c}\right)$ the Banach space of bounded analytic functions outside $E$ with supremum norm $\|\cdot\|_{H^{\infty}\left(E^{c}\right)}$. The analytic capacity of $E$ is defined by

$$
\gamma(E)=\sup \left\{\left|u^{\prime}(\infty)\right| ; u \in H^{\infty}\left(E^{c}\right),\|u\|_{H^{\infty}\left(E^{c}\right)} \leqslant 1\right\},
$$

where $u^{\prime}(\infty)=\lim _{z \rightarrow \infty} z(u(z)-u(\infty))[3$, p. 6]. We are concerned with estimating $\gamma(E)$. Let $D(z, r)$ denote the open disk of center $z$ and of radius $r$. For $\varepsilon>0$, we put $|E|_{(\varepsilon)}=2 \inf \sum_{k=1}^{\infty} r_{k}$, where the infimum is taken over all coverings $\left\{D\left(z_{k}, r_{k}\right)\right\}_{k=1}^{\infty}$ of $E$ with radii less than $\varepsilon$. The generalized length of $E$ is defined by $|E|=\lim _{\varepsilon \rightarrow 0}|E|_{(\varepsilon)}$; the limit exists since $|E|_{(\varepsilon)}$ is decreasing with respect to $\varepsilon$. It is well known that

$$
\gamma(E) \leqslant \text { Const }|E| \quad[3, \text { p. 48] }
$$

Let $\mathfrak{B}^{\theta}(-\pi / 2<\theta \leqslant \pi / 2)$ denote the straight line defined by the equation $x \sin \theta-y \cos \theta=0$ and let $\mathcal{L}(r, \theta)(r>0,-\pi<\theta \leqslant \pi)$ denote the straight line defined by the equation $x \cos \theta+y \sin \theta=r$. The Buffon length of $E$ is defined by

$$
\operatorname{Bu}(E)=\iint_{\{(r, \theta): \mathfrak{Q}(r, \theta) \cap E \neq \varnothing\}} d r d \theta .
$$

We easily see that $\operatorname{Bu}(E)=\int_{-\pi / 2}^{\pi / 2}\left|E^{\theta}\right| d \theta$, where $E^{\theta}$ is the projection of $E$ to $\mathfrak{B}^{\theta}$. If $E$ is contained in $D(0,1), \mathrm{Bu}(E) / 2 \pi$ is called the Buffon needle probability; this gives probability (measured by $\left.\left.d r\right|_{(0,1)} d \theta / 2 \pi\right)$ of straight lines $\mathfrak{L}(r, \theta)$ intersecting with $E$.

Suppose that the boundary $\partial E$ of $E$ consists of a finite number of rectifiable Jordan curves. We put

$$
\operatorname{Cr}(E)=\iint_{r>0,|\theta| \leqslant \pi} n(r, \theta) d r d \theta
$$

where $n(r, \theta)$ is the cardinal number of $\mathfrak{L}(r, \theta) \cap \partial E$. Then we have evidently $\operatorname{Bu}(E) \leqslant \operatorname{Cr}(E)$. Crofton's formula [8, p. 13] shows that $\operatorname{Cr}(E)=2|\partial E|$. Since

Received by the editors May 19, 1986.

1980 Mathematics Subject Classification. Primary 30C85.

Key words and phrases. Analytic capacity, the Buffon needle probability.

()1987 American Mathematical Society $0002-9947 / 87 \$ 1.00+\$ .25$ per page 
$\gamma(E)=\gamma(\partial E)$, (1) implies that $\gamma(E) \leqslant$ Const $\operatorname{Cr}(E)$. Thus both $\gamma(E)$ and $\operatorname{Bu}(E)$ are dominated by Const $\operatorname{Cr}(E)$. From this point of view, Ivanov [4] and Marshall [6] asked whether $\gamma(\cdot)$ and $\mathrm{Bu}(\cdot)$ are comparable or not. As an answer to this question, we show in this note

THEOREM. There exists a sequence $\left(E_{n}\right)_{n=1}^{\infty}$ of compact sets in $D(0,2)$ such that $\lim _{n \rightarrow \infty} \operatorname{Bu}\left(E_{n}\right) / \gamma\left(E_{n}\right)^{2-\varepsilon}=0$ for any $\varepsilon>0$.

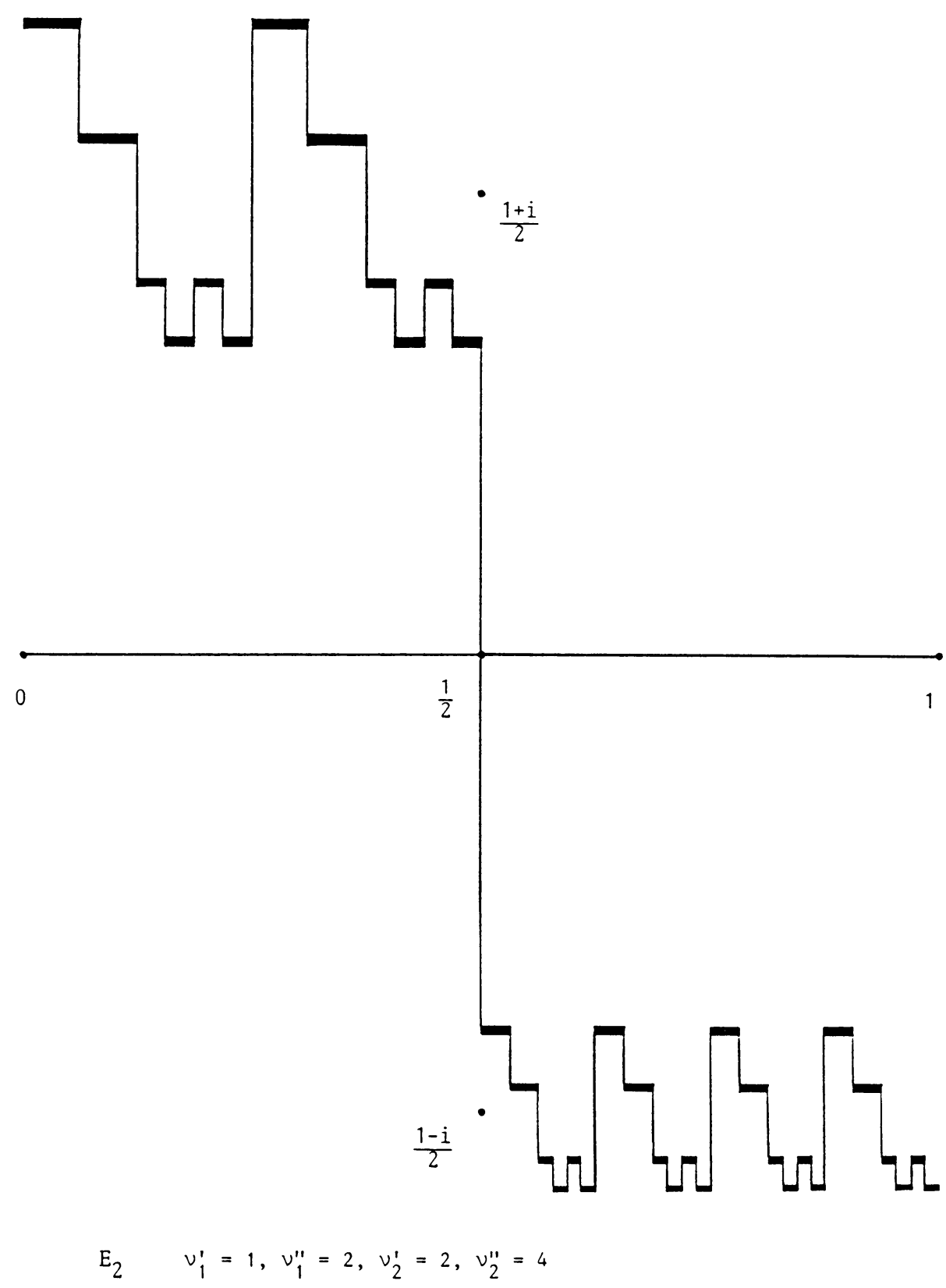

FIGURE 1 
The set $E_{n}$ which will be constructed later has a form like a crank with many pedals (see Figure 1). Our theorem shows that the inequality " $\gamma(\cdot) \leqslant \mathrm{Const} \mathrm{Bu}(\cdot)$ " is not valid. To prove our theorem, we remark

Proposition. Let $E$ be a compact set on a rectifiable graph $\Gamma=\{x+i A(x)$; $\alpha \leqslant x \leqslant \beta\}$. Then $\gamma(E) \geqslant$ Const $\left|E^{0}\right|^{3 / 2} /|\Gamma|^{1 / 2}$.

We do not know whether these exponents $3 / 2$ and $1 / 2$ are sharp or not. To prove our proposition, we shall use an estimate of the norm of the Calderon-Cauchy operator [7].

2. Proof of Proposition. Let $L^{p}(1 \leqslant p \leqslant \infty)$ denote the $L^{p}$ space on the real line R. Its norm is denoted by $\|\cdot\|_{p}$. For a real-valued function $b \in L^{\infty}$, we define a kernel by

$$
C[b](x, y)=1 /\{(x-y)+i(B(x)-B(y))\},
$$

where $B(x)=\int_{0}^{x} b(t) d t$. The singular integral operator $C[b]$ defined by the above kernel is called the Calderón-Cauchy operator. We denote by $\|C[b]\|_{2,2}$ the norm of $C[b]$ as an operator from $L^{2}$ to itself. We use an auxiliary operator $C^{*}[b]$ defined by

where

$$
C^{*}[b] f(x)=\sup _{\varepsilon>0}\left|C_{\varepsilon}[b] f(x)\right|,
$$

$$
C_{\varepsilon}[b] f(x)=\int_{|x-y|>\varepsilon} C[b](x, y) f(y) d y .
$$

The following fact is already known.

Lemma 1 [7]. $\|C[b]\|_{2,2} \leqslant \operatorname{Const}\left(1+\sqrt{\|b\|_{\infty}}\right)$.

In the same manner as Lemma 2 in [1, p. 139], the separation theorem yields

Lemma 2. Let $T$ be an operator defined by a kernel $T(x, y)$ satisfying $T(x, y)=$ $-T(y, x)(x, y \in \mathbf{R})$ and $\sup _{x, y \in \mathbf{R}}|T(x, y)|(1+|x-y|)<\infty$. Then, for any compact set $F \subset \mathbf{R}$, there exists a nonnegative function $f_{0} \in L^{\infty}$ such that

$$
\left\|T f_{0}\right\|_{\infty} \leqslant 1, \quad\left\|f_{0}\right\|_{\infty} \leqslant 1 /\|T\|_{w}, \quad\left\|f_{0}\right\|_{1} \geqslant|F| / 10\|T\|_{w}, \quad \operatorname{supp}\left(f_{0}\right) \subset F,
$$

where $\operatorname{supp}\left(f_{0}\right)$ is the support of $f_{0}$ and $\|T\|_{w}$ is the weak-(1,1) norm of $T$, that is,

$$
\|T\|_{w}=\sup \left\{|x \in \mathbf{R} ;| T f(x)|>1| /\|f\|_{1} ; f \in L^{1}\right\} .
$$

Lemma 3. $\left\|C^{*}[b]\right\|_{w} \leqslant \operatorname{Const}\left(1+\sqrt{\|b\|_{\infty}}\right)$.

Proof. Let $\omega(C[b])$ denote the minimum of all $M$ satisfying the following three inequalities:

$$
\begin{aligned}
& |C[b](x, y)| \leqslant M /|x-y| . \\
& \left|C[b](x, y)-C[b]\left(x^{\prime}, y\right)\right| \leqslant M\left|x-x^{\prime}\right|^{1 / 2} /|x-y|^{3 / 2} \quad\left(\left|x-x^{\prime}\right| \leqslant|x-y| / 2\right) . \\
& \left|C[b](x, y)-C[b]\left(x, y^{\prime}\right)\right| \leqslant M\left|y-y^{\prime}\right|^{1 / 2} /|x-y|^{3 / 2} \quad\left(\left|y-y^{\prime}\right| \leqslant|x-y| / 2\right) .
\end{aligned}
$$

We put $\|C[b]\|_{C Z}=\|C[b]\|_{2,2}+\omega(C[b])$. It is known that $\left\|C^{*}[b]\right\|_{w} \leqslant$ Const $\|C[b]\|_{C Z}[5$, Chapter 4$]$. Since $\omega(C[b]) \leqslant \operatorname{Const}\left(1+\sqrt{\|b\|_{\infty}}\right)$, Lemma 1 gives the required inequality. 
Lemma 4. For a compact set $F \subset \mathbf{R}$, there exists a nonnegative function $f_{F} \in L^{\infty}$ such that

$$
\begin{gathered}
\left\|C^{*}[b] f_{F}\right\|_{\infty} \leqslant 1, \quad\left\|f_{F}\right\|_{\infty} \leqslant 1 /\left(1+\sqrt{\|b\|_{\infty}}\right), \\
\left\|f_{F}\right\|_{1} \geqslant \text { Const }|F| /\left(1+\sqrt{\|b\|_{\infty}}\right), \quad \operatorname{supp}\left(f_{F}\right) \subset F .
\end{gathered}
$$

Proof. Lemma 3 shows that $\left\|C_{\varepsilon}[b]\right\|_{w} \leqslant \operatorname{Const}\left(1+\sqrt{\|b\|_{\infty}}\right) \quad(\varepsilon>0)$. Hence Lemma 2 shows that, to each $\varepsilon>0$, there corresponds a nonnegative function $f_{\varepsilon} \in L^{\infty}$ satisfying

$$
\begin{gathered}
\left\|C_{\varepsilon}[b] f_{\varepsilon}\right\|_{\infty} \leqslant 1, \quad\left\|f_{\varepsilon}\right\|_{\infty} \leqslant 1 /\left(1+\sqrt{\|b\|_{\infty}}\right), \\
\left\|f_{\varepsilon}\right\|_{1} \geqslant \text { Const }|F| /\left(1+\sqrt{\|b\|_{\infty}}\right) \text { and } \operatorname{supp}\left(f_{\varepsilon}\right) \subset F .
\end{gathered}
$$

Cotlar's inequality $[5$, p. 56] gives that

$$
\begin{aligned}
& \left|C_{\eta}[b] f_{\varepsilon}(x)\right| \leqslant \operatorname{Const}\left\{\mathfrak{m}\left(C_{\varepsilon}[b] f_{\varepsilon}\right)(x)+\|C[b]\|_{C Z} \mathfrak{m} f_{\varepsilon}(x)\right\} \\
& \qquad(x \in \mathbf{R}, 0<\varepsilon<\eta),
\end{aligned}
$$

where $m$ is the noncentered maximal operator $[5$, p. 9]. Hence

$$
\left\|C_{\eta}[b] f_{\varepsilon}\right\|_{\infty} \leqslant \text { Const }\left\{\left\|C_{\varepsilon}[b] f_{\varepsilon}\right\|_{\infty}+\|C[b]\|_{C Z}\left\|f_{\varepsilon}\right\|_{\infty}\right\} \leqslant \text { Const. }
$$

Let $f_{F}^{0}$ be a weak* cluster point of $\left(f_{\varepsilon}\right)_{\varepsilon>0}$ in the space of measures. Then we have $\left\|C_{\eta}[b] f_{F}^{0}\right\|_{\infty} \leqslant$ Const, which gives $\left\|C^{*}[b] f_{F}^{0}\right\|_{\infty} \leqslant$ Const. Multiplying $f_{F}^{0}$ by a suitable absolute constant, we obtain the required function $f_{F}$. Q.E.D.

LeMma 5. Let $F, f_{F}$ be the same as in Lemma 4 and let

$$
\hat{f}_{F}(z)=\int_{-\infty}^{\infty} \frac{f_{F}(y)}{\{z-(y+i B(y))\}} d y \quad(z \notin \hat{F}=\{x+i B(x) ; x \in F\}) .
$$

Then $\left\|\hat{f}_{F}\right\|_{H^{\infty}\left(\hat{F}^{\prime}\right)} \leqslant$ Const and $\left|\hat{f}_{F}^{\prime}(\infty)\right| \geqslant$ Const $|F| /\left(1+\sqrt{\|b\|_{\infty}}\right)$.

Proof. By Lemma 4, we have

$$
\left|\hat{f}_{F}^{\prime}(\infty)\right|=\left\|f_{F}\right\|_{1} \geqslant \text { Const }|F| /\left(1+\sqrt{\|b\|_{\infty}}\right) .
$$

We show $\left\|\hat{f}_{F}\right\|_{H^{\infty}\left(\hat{F}^{c}\right)} \leqslant$ Const. For any $z \notin \hat{F}$, we have

$$
\hat{f}_{F}(z)=\int_{-\infty}^{\infty} \frac{f_{F}(y)}{(\operatorname{Re} z-y)+i\{B(\operatorname{Re} z)-B(y)+(\operatorname{Im} z-B(\operatorname{Re} z))\}} d y
$$

as long as $\operatorname{Im} z \neq B(\operatorname{Re} z)$. Hence it is sufficient to show that

$$
\sup _{x, s \in \mathbf{R}, s \neq 0}\left|\int_{-\infty}^{\infty} \frac{f_{F}(y)}{(x-y)+i(B(x)-B(y)+s)} d y\right| \leqslant \text { Const. }
$$


Let $s_{0}=2\left(1+\|b\|_{\infty}\right)$. We have, for any $x, s \in \mathbf{R}, s \neq 0$,

$$
\begin{aligned}
& \left|\int_{-\infty}^{\infty} \frac{f_{F}(y)}{(x-y)+i(B(x)-B(y)+s)} d y\right| \\
& \leqslant\left|\int_{|x-y| \leqslant|s| / s_{0} \mid}\right|+\left|\int_{|s| / s_{0}<|x-y| \leqslant|s|}\right|+\left|\int_{|x-y|>|s|}\right| \\
& \leqslant \int_{|x-y| \leqslant|s| / s_{0}} \frac{f_{F}(y)}{|s|-|B(x)-B(y)|} d y+\int_{|s| / s_{0}<|x-y| \leqslant|s|} \frac{f_{F}(y)}{|x-y|} d y \\
& \quad+\left|\int_{|x-y|>|s|} \frac{s f_{F}(y)}{\{(x-y)+i(B(x)-B(y)+s)\}\{(x-y)+i(B(x)-B(y))\}} d y\right| \\
& \quad+\left|C_{|s|}[b] f_{F}(x)\right| \\
& \leqslant \text { Const }\left\|f_{F}\right\|_{\infty}+\left\|f_{F}\right\|_{\infty} \log s_{0}+\left\|f_{F}\right\|_{\infty}|s| \int_{|x-y|>|s|} \frac{f_{F}(y)}{(x-y)^{2}} d y+C^{*}[b] f_{F}(x) \\
& \leqslant \text { Const }\left(1+\log s_{0}\right)\left\|f_{F}\right\|_{\infty}+\left\|C^{*}[b] f_{F}\right\|_{\infty} \leqslant \text { Const. } \quad \text { Q.E.D. }
\end{aligned}
$$

LEMMA 6. Let $E$ and $\Gamma$ be the same as in the theorem. Then there exists a graph $\Gamma^{*}=\{x+i B(x) ; \quad \alpha \leqslant x \leqslant \beta\}, \quad B(x)=\int_{\alpha}^{x} b(t) d t+A(\alpha) ;$ such that $\|b\|_{\infty} \leqslant$ $4|\Gamma| /\left|E^{0}\right|$ and $|\alpha \leqslant x \leqslant \beta ; A(x) \neq B(x)| \leqslant\left|E^{0}\right| / 2$.

Proof. Let $t_{0}=2|\Gamma| /\left|E^{0}\right|$. Then

$$
t_{0} \geqslant \frac{|\Gamma|}{\beta-\alpha} \geqslant \frac{1}{\beta-\alpha} \int_{\alpha}^{\beta}|a(t)| d t \quad\left(a=A^{\prime}\right) .
$$

The Calderón-Zygmund decomposition [5, p. 12] shows that there exists a sequence $\left(I_{k}\right)_{k=1}^{\infty}$ of mutually nonoverlapping intervals in $[\alpha, \beta]$ such that

$$
\begin{gathered}
|a(x)| \leqslant t_{0} \quad \text { a.e. in }[\alpha, \beta]-\bigcup_{k=1}^{\infty} I_{k}, \\
t_{0} \leqslant \frac{1}{\left|I_{k}\right|} \int_{I_{k}}|a(t)| d t \leqslant 2 t_{0} \quad(k \geqslant 1) .
\end{gathered}
$$

We put

$$
b(x)= \begin{cases}a(x) & \left(x \in[\alpha, \beta]-\bigcup_{k=1}^{\infty} I_{k}\right), \\ \frac{1}{\left|I_{k}\right|} \int_{I_{k}} a(t) d t & \left(x \in I_{k}, k \geqslant 1\right) .\end{cases}
$$

Then $\|b\|_{\infty} \leqslant 2 t_{0}=4|\Gamma| /\left|E^{0}\right|$. We have, with $B(x)=\int_{\alpha}^{x} b(t) d t+A(\alpha)$,

$$
\begin{aligned}
|\alpha \leqslant x \leqslant \beta ; A(x) \neq B(x)| & \leqslant \sum_{k=1}^{\infty}\left|I_{k}\right| \leqslant \frac{1}{t_{0}} \sum_{k=1}^{\infty} \int_{I_{k}}|a(t)| d t \\
& \leqslant|\Gamma| / t_{0}=\left|E^{0}\right| / 2 .
\end{aligned}
$$

Thus $\Gamma^{*}=\{x+i B(x) ; \alpha \leqslant x \leqslant \beta\}$ satisfies the required two inequalities. Q.E.D. 
We now prove our proposition.Let $\Gamma^{*}$ be the graph given in Lemma 6 . We can choose a compact set $F$ in $E^{0} \cap\{\alpha \leqslant x \leqslant \beta ; A(x)=B(x)\}$ so that $|F| \geqslant\left|E^{0}\right| / 4$. Let $\hat{f}_{F}$ be the function in Lemma 5. Since $\hat{F} \subset E$, we have $\hat{f}_{F} \in H^{\infty}\left(E^{c}\right)$, $\left\|\hat{f}_{F}\right\|_{H^{\infty}\left(E^{\prime}\right)} \leqslant$ Const. Thus

$$
\begin{aligned}
\gamma(E) & \geqslant\left|\hat{f}_{F}^{\prime}(\infty)\right| \geqslant \text { Const }|F| /\left(1+\sqrt{\|b\|_{\infty}}\right) \\
& \geqslant \text { Const }|F| /\left\{1+\sqrt{4|\Gamma| /\left|E^{0}\right|}\right\} \geqslant \text { Const }\left|E^{0}\right|^{3 / 2} /|\Gamma|^{1 / 2} .
\end{aligned}
$$

This completes the proof of our proposition.

3. Construction of $\left(E_{n}\right)$. In this section we construct a sequence $\left(E_{n}\right)_{n=2}^{\infty}$ of compact sets in $D(0,2)$ so that

$$
\begin{gathered}
\gamma\left(E_{n}\right) \geqslant \text { Const } / \sqrt{n}, \\
\mathrm{Bu}\left(E_{n}\right) \leqslant \operatorname{Const}(\log n)^{2} / n .
\end{gathered}
$$

If such a sequence has been constructed, the assertion of our theorem is evidently deduced. Let $I_{0}=[0,1]$ and $U=I_{0} \times[-1,1]$. For two sets $G, G^{\prime}$ in $U$ and a positive integer $\nu$, we define

$$
\sigma\left(G, G^{\prime}\right)=\left(\frac{1}{2} G+\frac{i}{2}\right) \cup\left(\frac{1}{2} G^{\prime}+\frac{1-i}{2}\right), \quad \tau(\nu, G)=\bigcup_{\mu=0}^{\nu-1}\left(\frac{1}{\nu} G+\frac{\mu}{\nu}\right),
$$

where $\zeta G+\zeta^{\prime}=\left\{\zeta z+\zeta^{\prime} ; z \in G\right\}\left(\zeta, \zeta^{\prime} \in \mathbf{C}\right)$. Given $n \geqslant 2$, we shall define two finite increasing sequences $\left(\nu_{k}^{\prime}\right)_{k=1}^{n},\left(\nu_{k}^{\prime \prime}\right)_{k=1}^{n}$ of positive integers later. To these two sequences, we associate $n+1$ sets $G_{0}, G_{1}, \ldots, G_{n}$ as follows:

$$
G_{0}=\sigma\left(I_{0}, I_{0}\right), \quad G_{k}=\sigma\left(\tau\left(v_{k}^{\prime}, G_{k-1}\right), \tau\left(\nu_{k}^{\prime \prime}, G_{k-1}\right)\right) \quad(1 \leqslant k \leqslant n) .
$$

We put $E_{n}=G_{n}$ (see Figure 1).

Here is a lemma necessary for the proof of (2).

Lemma 7. There exists a graph $\Gamma_{n}=\left\{x+i A_{n}(x) ; 0 \leqslant x \leqslant 1\right\}$ such that $\left|\Gamma_{n}\right| \leqslant$ Const $n$ and $\left|\left(\Gamma_{n} \cap E_{n}\right)^{0}\right| \geqslant \frac{1}{2}$.

Proof. For two sets $G, G^{\prime}$ in $U$, we put

$$
\tilde{\sigma}\left(G, G^{\prime}\right)=\sigma\left(G, G^{\prime}\right) \cup\left(\frac{i}{2} I_{0}\right) \cup\left(i I_{0}+\frac{1-i}{2}\right) \cup\left(-\frac{i}{2} I_{0}+1\right) .
$$

Then

$$
\left|\tilde{\sigma}\left(G, G^{\prime}\right)\right| \leqslant\left|\sigma\left(G, G^{\prime}\right)\right|+2 \leqslant \frac{1}{2}\left(|G|+\left|G^{\prime}\right|\right)+2 .
$$

For a positive integer $\nu$ and a set $G$ in $U$, we have $|\tau(\nu, G)| \leqslant|G|$. We now define $n+1$ arcwise connected sets $\Lambda_{0}, \Lambda_{1}, \ldots, \Lambda_{n}$ by

$$
\Lambda_{0}=\tilde{\sigma}\left(I_{0}, I_{0}\right), \quad \Lambda_{k}=\tilde{\sigma}\left(\tau\left(v_{k}^{\prime}, \Lambda_{k-1}\right), \tau\left(\nu_{k}^{\prime \prime}, \Lambda_{k-1}\right)\right) \quad(1 \leqslant k \leqslant n) .
$$

Then we have $\Lambda_{n} \supset E_{n}$ and

$$
\begin{aligned}
\left|\Lambda_{n}\right| & \leqslant \frac{1}{2}\left(\left|\tau\left(v_{n}^{\prime}, \Lambda_{n-1}\right)\right|+\left|\tau\left(v_{n}^{\prime \prime}, \Lambda_{n-1}\right)\right|\right)+2 \\
& \leqslant\left|\Lambda_{n-1}\right|+2 \leqslant \cdots \leqslant\left|\Lambda_{0}\right|+2 n=2 n+3 .
\end{aligned}
$$


We see that $\Lambda_{n} \cap \mathfrak{Q}(r, 0)$ is a singleton except for $r>0$ such that $\mathfrak{L}(r, 0)$ passes through one of the endpoints of $E_{n}$. Hence we can define a graph $\Gamma_{n}$ satisfying the required two inequalities. Q.E.D.

We now show (2). Let $\Gamma_{n}$ be the graph given in Lemma 7. We put $\tilde{E}_{n}=E_{n} \cap \Gamma_{n}$. Then our proposition shows that

$$
\begin{aligned}
\gamma\left(E_{n}\right) & \geqslant \gamma\left(\tilde{E}_{n}\right) \geqslant \text { Const }\left|\tilde{E}_{n}^{0}\right|^{3 / 2} /\left|\Gamma_{n}\right|^{1 / 2} \\
& \geqslant \text { Const } /\left|\Gamma_{n}\right|^{1 / 2} \geqslant \text { Const } / \sqrt{n} .
\end{aligned}
$$

Thus we have (2).

4. Proof of (3). In this section we define $\left(\nu_{k}^{\prime}\right)_{k=1}^{n},\left(\nu_{k}^{\prime \prime}\right)_{k=1}^{n}$ such that (3) holds. Geometric observation immediately yields

Lemma 8. For $0 \leqslant k \leqslant n, G_{k}$ is a union of $\Pi_{j=0}^{k}\left(\nu_{j}^{\prime}+\nu_{j}^{\prime \prime}\right)$ segments in $U$ $\left(\nu_{0}^{\prime}=\nu_{0}^{\prime \prime}=1\right)$.

Lemma 9. Let $X$ be a union of at most $\nu$ intervals in an interval $I=[s, s+r]$ on $\mathbf{R}$ and let $Y$ be a set in $I$ of the form $Y=\bigcup_{j=0}^{\mu^{\prime \prime}-1}\left(Y^{\prime}+\left(j r / \mu^{\prime \prime}\right)\right)$ for some $\mu^{\prime \prime} \geqslant 1$ and $Y^{\prime} \subset\left[s, s+\left(r / \mu^{\prime \prime}\right)\right]$. Then

$$
|X \cup Y| \leqslant|X|+|Y|-|X||Y| / r+3 r \nu / \mu^{\prime \prime} .
$$

PRoof. We can write $X=\bigcup_{j=1}^{\mu^{\prime}} J_{j}$ with mutually disjoint intervals $\left\{J_{j}\right\}_{j=1}^{\mu^{\prime}}, \mu^{\prime} \leqslant \nu$. Then, for each $J_{j}$, the number of $k$ 's satisfying $\left(s+\left(k r / \mu^{\prime \prime}\right), s+\left\{(k+1) r / \mu^{\prime \prime}\right\}\right)$ $\subset J_{j}$ is larger than $\left(\mu^{\prime \prime}\left|J_{j}\right| / r\right)-3$. Hence

$$
\begin{aligned}
|X \cap Y| & =\sum_{j=1}^{\mu^{\prime}}\left|J_{j} \cap Y\right| \geqslant \sum_{j=1}^{\mu^{\prime}}\left|Y^{\prime}\right|\left\{\frac{\mu^{\prime \prime}\left|J_{j}\right|}{r}-3\right\} \\
& =\frac{\mu^{\prime \prime}\left|Y^{\prime}\right|}{r} \sum_{j=1}^{\mu^{\prime}}\left|J_{j}\right|-3 \mu^{\prime}\left|Y^{\prime}\right| \geqslant \frac{|X||Y|}{r}-\frac{3 r \nu}{\mu^{\prime \prime}},
\end{aligned}
$$

which gives the required inequality. Q.E.D.

For $z \in \mathbf{C}$ and $\xi \in \mathbf{R}$, we write $x(z, \xi)=\operatorname{Re} z+\xi \operatorname{Im} z$. The straight line passing through $z$ and $x(z, \xi)$ is perpendicular to $\mathfrak{B}^{\arctan \xi}$. For a set $G \subset \mathbf{C}$, we write $G(\xi)=\{x(z, \xi) ; z \in G\}$. Then $(s G+\zeta)(\xi)=s G(\xi)+x(\zeta, \xi)(s \in \mathbf{R}, \zeta \in \mathbf{C})$.

LEMMA 10. Let $G$ be a set in $U$ with at most $\nu$ components. For three nonnegative integers $\nu^{\prime}, \nu^{\prime \prime}, m$ with $\nu^{\prime \prime} \geqslant \nu^{\prime} \geqslant m+1$, we put

$$
H=H\left(\nu^{\prime}, \nu^{\prime \prime}, m, G\right)=\left(\frac{1}{2} \tau\left(\nu^{\prime}, G\right)+i / 2\right) \cup\left(\frac{1}{2} \tau\left(\nu^{\prime \prime}, G\right)+(1-i) / 2+m\right) .
$$

Let $m \leqslant \xi \leqslant m+1$. Then

$$
|H(\xi)| \leqslant\left\{1-\xi_{m}|G(\xi)|\right\}|G(\xi)|+\operatorname{Const}\left\{(m+1) / \nu^{\prime}+\nu \nu^{\prime} / \nu^{\prime \prime}\right\}
$$

as long as $|G(\xi)| \leqslant 1$, where $\xi_{m}=\min \{\xi-m, m+1-\xi\}$. 
Proof. Suppose that $m \leqslant \xi \leqslant m+\frac{1}{2}$. We put

$$
\begin{gathered}
X_{j}=\frac{1}{2 \nu^{\prime}} G+\frac{j}{2 \nu^{\prime}}+\frac{i}{2} \quad\left(0 \leqslant j \leqslant \nu^{\prime}-1\right), \\
Y_{j}=\frac{1}{2 \nu^{\prime \prime}} G+\frac{j}{2 \nu^{\prime \prime}}+\frac{1-i}{2}+m \quad(j=0, \pm 1, \pm 2, \ldots) .
\end{gathered}
$$

Then $\left|X_{j}(\xi)\right|=|G(\xi)| / 2 \nu^{\prime}, \quad\left|Y_{j}(\xi)\right|=|G(\xi)| / 2 \nu^{\prime \prime}$. Let $\mu^{\prime}=\iota\left(2 \nu^{\prime} \xi_{m}\right)$ and $\mu^{\prime \prime}=$ $\iota\left(2 \nu^{\prime \prime} \xi_{m}\right)$, where $\iota(x)$ is the integral part of $x$. If $\mu^{\prime} \leqslant 2 m+2$, then, by $2 \nu^{\prime}\left|X_{j}(\xi)\right|=$ $2 \nu^{\prime \prime}\left|Y_{j}(\xi)\right|=|G(\xi)| \leqslant 1$, we have

$$
\begin{aligned}
|H(\xi)| & =\left|\left(\frac{1}{2} \tau\left(\nu^{\prime}, G\right)+\frac{i}{2}\right)(\xi) \cup\left(\frac{1}{2} \tau\left(\nu^{\prime \prime}, G\right)+\frac{1-i}{2}+m\right)(\xi)\right| \\
& =\left|\left\{\bigcup_{j=0}^{\nu^{\prime}-1} X_{j}(\xi)\right\} \cup\left\{\bigcup_{j=0}^{\nu^{\prime \prime}-1} Y_{j}(\xi)\right\}\right| \\
& \leqslant \sum_{j=0}^{\nu^{\prime}-1}\left|X_{j}(\xi)\right|+\sum_{j=0}^{\nu^{\prime \prime}-1}\left|Y_{j}(\xi)\right|=|G(\xi)| \\
& \leqslant|G(\xi)|+\left\{\frac{\mu^{\prime}+1}{2 \nu^{\prime}}-\xi_{m}\right\} \leqslant|G(\xi)|+\left\{\frac{\mu^{\prime}+1}{2 \nu^{\prime}}-\xi_{m}|G(\xi)|^{2}\right\} \\
& \leqslant\left\{1-\xi_{m}|G(\xi)|\right\}|G(\xi)|+\frac{2 m+3}{2 \nu^{\prime}},
\end{aligned}
$$

which gives the required inequality. Suppose that $\mu^{\prime} \geqslant 2 m+3$. We apply Lemma 9 to

$$
\begin{gathered}
X=\bigcup_{j=\nu^{\prime}-\mu^{\prime}+m+1}^{\nu^{\prime}-m-2} X_{j}(\xi), \quad Y=\left\{\bigcup_{j=-\infty}^{\infty} Y_{j}(\xi)\right\} \cap I, \\
I=\left[x((1-i) / 2+m, \xi), x\left(\mu^{\prime \prime} / 2 \nu^{\prime \prime}+(1-i) / 2+m, \xi\right)\right] ;
\end{gathered}
$$

geometric observation shows that $X \subset I$ and $Y=\bigcup_{j=0}^{\mu^{\prime \prime}-1}\left(Y^{\prime}+\left(j|I| / \mu^{\prime \prime}\right)\right)$ with

$$
Y^{\prime}=Y \cap\left[x((1-i) / 2+m, \xi), x((1-i) / 2+m, \xi)+|I| / \mu^{\prime \prime}\right]
$$

(see Figure 2). Since $\mu^{\prime} \leqslant \nu^{\prime},|I|=\mu^{\prime \prime} / 2 \nu^{\prime \prime}$ and $X$ is a union of at most $\nu \mu^{\prime}$ intervals, Lemma 9 gives that

$$
\begin{aligned}
|X \cup Y| & \leqslant|X|+|Y|-|X||Y| /|I|+3 \nu \mu^{\prime}|I| / \mu^{\prime \prime} \\
& \leqslant|Y|+|X|\{1-|Y| /|I|\}+2 \nu \nu^{\prime} / \nu^{\prime \prime} .
\end{aligned}
$$

Since $|G(\xi)| \leqslant 1,|X| \leqslant\left(\mu^{\prime}-2 m-2\right)|G(\xi)| / 2 v^{\prime}$,

$$
\frac{\mu^{\prime}-2 m-2}{2 \nu^{\prime}} \frac{|G(\xi)|}{|I|} \leqslant \frac{\xi_{m}-1 / 2 \nu^{\prime}}{|I|} \leqslant \frac{\mu^{\prime \prime}+1 / 2 \nu^{\prime \prime}-1 / 2 \nu^{\prime}}{|I|} \leqslant \frac{\mu^{\prime \prime}}{2 \nu^{\prime \prime}|I|}=1
$$


and

$$
|Y|=\left|\left\{\bigcup_{j=-m-1}^{\mu^{\prime \prime}+m} Y_{j}(\xi)\right\} \cap I\right| \leqslant \frac{\mu^{\prime \prime}+2 m+2}{2 \nu^{\prime \prime}}|G(\xi)|
$$

we have

$$
\begin{aligned}
& |X \cup Y| \leqslant|Y|+\left(\frac{\mu^{\prime}-2 m-2}{2 \nu^{\prime}}|G(\xi)|\right)\left\{1-\frac{|Y|}{|I|}\right\}+\frac{2 \nu \nu^{\prime}}{\nu^{\prime \prime}} \\
& =\frac{\mu^{\prime}-2 m-2}{2 \nu^{\prime}}|G(\xi)|+|Y|\left\{1-\frac{\mu^{\prime}-2 m-2}{2 \nu^{\prime}} \frac{|G(\xi)|}{|I|}\right\}+\frac{2 \nu \nu^{\prime}}{\nu^{\prime \prime}} \\
& \leqslant \frac{\mu^{\prime}-2 m-2}{2 \nu^{\prime}}|G(\xi)| \\
& +\left(\frac{\mu^{\prime \prime}+2 m+2}{2 \nu^{\prime \prime}}|G(\xi)|\right)\left\{1-\frac{\mu^{\prime}-2 m-2}{2 \nu^{\prime}} \frac{|G(\xi)|}{|I|}\right\}+\frac{2 \nu \nu^{\prime}}{\nu^{\prime \prime}} \\
& \leqslant \frac{\mu^{\prime}-2 m-2}{2 \nu^{\prime}}|G(\xi)| \\
& +\frac{\mu^{\prime \prime}}{2 \nu^{\prime \prime}}|G(\xi)|\left\{1-\frac{\mu^{\prime}-2 m-2}{2 \nu^{\prime}} \frac{|G(\xi)|}{|I|}\right\}+\left\{\frac{m+1}{\nu^{\prime \prime}}+\frac{2 \nu \nu^{\prime}}{\nu^{\prime \prime}}\right\} \\
& =\left\{\frac{\mu^{\prime}-2 m-2}{2 \nu^{\prime}}+\frac{\mu^{\prime \prime}}{2 \nu^{\prime \prime}}\right\}|G(\xi)|-\frac{\mu^{\prime}-2 m-2}{2 \nu^{\prime}}|G(\xi)|^{2}+\left\{\frac{m+1}{\nu^{\prime \prime}}+\frac{2 \nu \nu^{\prime}}{\nu^{\prime \prime}}\right\} \\
& \leqslant\left\{\frac{\mu^{\prime}-2 m-2}{2 \nu^{\prime}}+\frac{\mu^{\prime \prime}}{2 \nu^{\prime \prime}}\right\}|G(\xi)|-\xi_{m}|G(\xi)|^{2}+\left\{\frac{2 m+3}{2 \nu^{\prime}}+\frac{m+1}{\nu^{\prime \prime}}+\frac{2 \nu \nu^{\prime}}{\nu^{\prime \prime}}\right\} \text {. }
\end{aligned}
$$
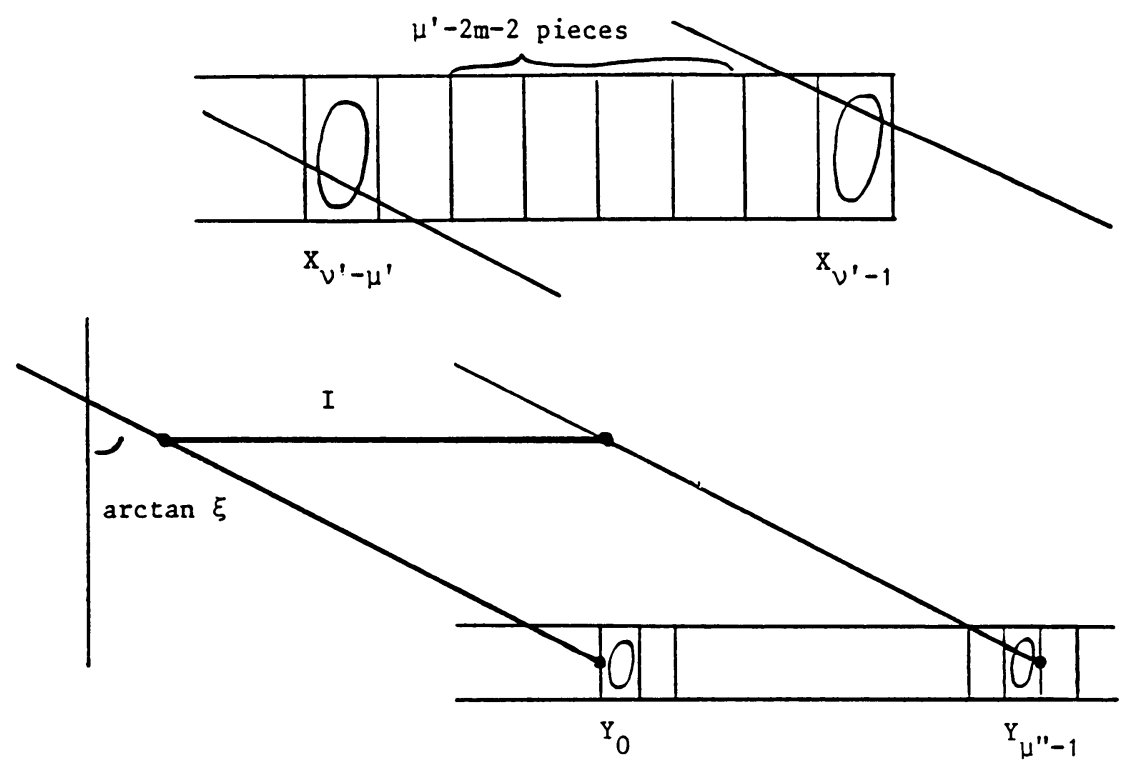

FIGURE 2 
Thus

$$
\begin{aligned}
|H(\xi)| & =\left|\left\{\bigcup_{j=0}^{\nu^{\prime}-1} X_{j}(\xi)\right\} \cup\left\{\bigcup_{j=0}^{\nu^{\prime \prime}-1} Y_{j}(\xi)\right\}\right| \\
& \leqslant\left|\left\{\bigcup_{j=0}^{\nu^{\prime}-1} X_{j}(\xi)-X\right\} \cup\left[\left\{\bigcup_{j=0}^{m}+\sum_{j=\mu^{\prime \prime}-m-1}^{\nu^{\prime \prime}-1}\right\} Y_{j}(\xi)\right] \cup(X \cup Y)\right| \\
& \leqslant\left\{\sum_{j=0}^{\nu^{\prime}-\mu^{\prime}+m}+\sum_{j=\nu^{\prime}-m-1}^{\nu^{\prime}-1}\right\}\left|X_{j}(\xi)\right|+\left\{\sum_{j=0}^{m}+\sum_{j=\mu^{\prime \prime}-m-1}^{\nu^{\prime \prime}-1}\right\}\left|Y_{j}(\xi)\right|+|X \cup Y| \\
& =\left\{\frac{\nu^{\prime}-\mu^{\prime}+2 m+2}{2 \nu^{\prime}}+\frac{\nu^{\prime \prime}-\mu^{\prime \prime}}{2 \nu^{\prime \prime}}\right\}|G(\xi)|+|X \cup Y|+\frac{2 m+2}{2 \nu^{\prime \prime}}|G(\xi)| \\
& \leqslant\left\{1-\xi_{m}|G(\xi)|\right\}|G(\xi)|+\text { Const }\left\{\frac{m+1}{\nu^{\prime}}+\frac{\nu \nu^{\prime}}{\nu^{\prime \prime}}\right\} .
\end{aligned}
$$

In the case where $m+\frac{1}{2}<\xi \leqslant m+1$, we discuss $H(\xi)$ on an interval

$$
\left[x\left(-\frac{\iota\left(2 \nu^{\prime \prime} \xi_{m}\right)}{2 \nu^{\prime \prime}}+\frac{1-i}{2}+m+\frac{1}{2}, \xi\right), x\left(\frac{1-i}{2}+m+\frac{1}{2}, \xi\right)\right] .
$$

Then, in the same manner as above, we obtain the required inequality. Q.E.D.

Lemma 11. $\left|G_{k}(\xi)\right| \leqslant\left|G_{k-1}(\xi)\right| \leqslant 1 \quad(\xi \in \mathbf{R}, 1 \leqslant k \leqslant n)$.

Proof. We easily see that $\left|G_{0}(\xi)\right| \leqslant 1$. We have

$$
\begin{aligned}
\left|G_{k}(\xi)\right| & =\left|\sigma\left(\tau\left(\nu_{k}^{\prime}, G_{k-1}\right), \tau\left(\nu_{k}^{\prime \prime}, G_{k-1}\right)\right)(\xi)\right| \\
& =\left|\left(\frac{1}{2} \tau\left(\nu_{k}^{\prime}, G_{k-1}\right)(\xi)+\frac{\xi}{2}\right) \cup\left(\frac{1}{2} \tau\left(\nu_{k}^{\prime \prime}, G_{k-1}\right)(\xi)+\frac{1-\xi}{2}\right)\right| \\
& \leqslant \frac{1}{2}\left\{\left|\tau\left(\nu_{k}^{\prime}, G_{k-1}\right)(\xi)\right|+\left|\tau\left(\nu_{k}^{\prime \prime}, G_{k-1}\right)(\xi)\right|\right\} \\
& =\frac{1}{2}\left\{\left|\bigcup_{j=0}^{\nu_{k}^{\prime}-1}\left(\frac{1}{\nu_{k}^{\prime}} G_{k-1}(\xi)+\frac{j}{\nu_{k}^{\prime}}\right)\right|+\left|\bigcup_{j=0}^{\nu_{k}^{\prime \prime}-1}\left(\frac{1}{\nu_{k}^{\prime \prime}} G_{k-1}(\xi)+\frac{j}{\nu_{k}^{\prime \prime}}\right)\right|\right\} \\
& \leqslant\left|G_{k-1}(\xi)\right| . \text { Q.E.D. }
\end{aligned}
$$

We now define $\left(\nu_{k}^{\prime}\right)_{k=1}^{n},\left(\nu_{k}^{\prime \prime}\right)_{k=1}^{n}$ such that (3) holds. We see that $\left|E_{n}^{\theta}\right|=$ $\left|E_{n}(\tan \theta)\right| \cos \theta(-\pi / 2<\theta \leqslant \pi / 2)$. Hence we have

$$
\begin{aligned}
\operatorname{Bu}\left(E_{n}\right) & =\int_{-\pi / 2}^{\pi / 2}\left|E_{n}^{\theta}\right| d \theta=\int_{-\infty}^{\infty} \frac{\left|E_{n}(\xi)\right|}{\left(1+\xi^{2}\right)^{3 / 2}} d \xi \\
& =\sum_{m=-n}^{n} \int_{m}^{m+1}+\int_{-\infty}^{-n}+\int_{n+1}^{\infty}\left(=\sum_{m=-n}^{n} \delta_{m}+\delta_{-n-1}+\delta_{n+1}, \text { say }\right) .
\end{aligned}
$$


We first estimate $\delta_{0}$. Recall that $G_{k-1}$ is a union of $\prod_{j=0}^{k-1}\left(\nu_{j}^{\prime}+\nu_{j}^{\prime \prime}\right)$ segments in $U$. Applying Lemma 10 to $G=G_{k-1}, \nu^{\prime}=\nu_{k}^{\prime}, \nu^{\prime \prime}=\nu_{k}^{\prime \prime}, m=0$, we have, for any $0 \leqslant \xi<1$,

$$
\begin{aligned}
\left|G_{k}(\xi)\right| & =\left|\sigma\left(\tau\left(\nu_{k}^{\prime}, G_{k-1}\right), \tau\left(\nu_{k}^{\prime \prime}, G_{k-1}\right)\right)(\xi)\right|=\left|H\left(\nu_{k}^{\prime}, \nu_{k}^{\prime \prime}, 0, G_{k-1}\right)(\xi)\right| \\
& \leqslant\left\{1-\xi_{0}\left|G_{k-1}(\xi)\right|\right\}\left|G_{k-1}(\xi)\right|+\text { Const }\left[\frac{1}{\nu_{k}^{\prime}}+\left\{\prod_{j=0}^{k-1}\left(\nu_{j}^{\prime}+v_{j}^{\prime \prime}\right)\right\} \frac{\nu_{k}^{\prime}}{\nu_{k}^{\prime \prime}}\right] .
\end{aligned}
$$

Since this estimate is valid for any $1 \leqslant k \leqslant n$, we obtain

(4)

$$
\begin{aligned}
& \left|E_{n}(\xi)\right|=\left|G_{n}(\xi)\right| \\
& \leqslant \prod_{k=1}^{n}\left\{1-\xi_{0}\left|G_{k-1}(\xi)\right|\right\}\left|G_{0}(\xi)\right|+C_{0} \sum_{k=1}^{n}\left[\frac{1}{v_{k}^{\prime}}+\left\{\prod_{j=0}^{k-1}\left(\nu_{j}^{\prime}+\nu_{j}^{\prime \prime}\right)\right\} \frac{v_{k}^{\prime}}{v_{k}^{\prime \prime}}\right] \\
& \quad \leqslant\left\{1-\xi_{0}\left|E_{n}(\xi)\right|\right\}^{n}+C_{0} \rho_{n},
\end{aligned}
$$

by Lemma 11 , where $C_{0}$ is an absolute constant and

$$
\rho_{n}=\sum_{k=1}^{n}\left[\frac{n}{\nu_{k}^{\prime}}+\left\{\prod_{j=0}^{k-1}\left(\nu_{j}^{\prime}+\nu_{j}^{\prime \prime}\right)\right\} \frac{\nu_{k}^{\prime}}{\nu_{k}^{\prime \prime}}\right] .
$$

We define $\xi_{0}^{\prime}$ by $1 / \xi_{0}^{\prime}=1 / \xi_{0}+C_{0} n \rho_{n}$. If $\left|E_{n}(\xi)\right|>\left(1 / n \xi_{0}^{\prime}\right) \log \left(10+n \xi_{0}^{\prime}\right)$, then (4) shows that

$$
\begin{aligned}
\left|E_{n}(\xi)\right| & \leqslant\left\{1-\xi_{0} \frac{1}{n \xi_{0}^{\prime}} \log \left(10+n \xi_{0}^{\prime}\right)\right\}^{n}+C_{0} \rho_{n} \\
& \leqslant\left\{1-\frac{1}{n} \log \left(n \xi_{0}^{\prime}\right)\right\}^{n}+C_{0} \rho_{n} \\
& \leqslant \frac{1}{n \xi_{0}^{\prime}}+C_{0} \rho_{n} \leqslant \frac{2}{n \xi_{0}^{\prime}} \leqslant \frac{1}{n \xi_{0}^{\prime}} \log \left(10+n \xi_{0}^{\prime}\right)<\left|E_{n}(\xi)\right|,
\end{aligned}
$$

which is a contradiction. Thus (4) yields that $\left|E_{n}(\xi)\right| \leqslant\left(1 / n \xi_{0}^{\prime}\right) \log \left(10+n \xi_{0}^{\prime}\right)(0 \leqslant \xi$ $\leqslant 1)$. We have

$$
\begin{aligned}
\delta_{0} & \leqslant \int_{0}^{1}\left|E_{n}(\xi)\right| d \xi \leqslant \frac{2}{n}+\int_{1 / n}^{1-1 / n} \frac{1}{n \xi_{0}^{\prime}} \log \left(10+n \xi_{0}^{\prime}\right) d \xi \\
& \leqslant \frac{2}{n}+2 \int_{1 / n}^{1 / 2}\left(\frac{1}{n \xi}+C_{0} \rho_{n}\right) \log (10+n \xi) d \xi \\
& \leqslant \operatorname{Const}\left\{\frac{(\log n)^{2}}{n}+\rho_{n} \log n\right\} .
\end{aligned}
$$

We next estimate $\delta_{m}(1 \leqslant m \leqslant n)$. We have, for any $2 \leqslant k \leqslant n, m \leqslant \xi \leqslant m+1$,

$$
\left|G_{k}(\xi)\right| \leqslant \frac{1}{2}\left\{\left|\tau\left(\nu_{k}^{\prime}, G_{k-1}\right)(\xi)\right|+\left|\tau\left(\nu_{k}^{\prime \prime}, G_{k-1}\right)(\xi)\right|\right\} .
$$


To estimate $\left|\tau\left(\nu_{k}^{\prime}, G_{k-1}\right)(\xi)\right|$, we put

$$
\begin{aligned}
X_{j}^{*}=\frac{1}{\nu_{k}^{\prime}}\left(\frac{1}{2} X^{*}+\frac{i}{2}\right)+\frac{j}{\nu_{k}^{\prime}}, \quad Y_{j}^{*}=\frac{1}{\nu_{k}^{\prime}}\left(\frac{1}{2} Y^{*}+\frac{1-i}{2}\right)+\frac{j}{\nu_{k}^{\prime}} & \left(0 \leqslant j \leqslant \nu_{k}^{\prime}-1\right),
\end{aligned}
$$

where $X^{*}=\tau\left(\nu_{k-1}^{\prime}, G_{k-2}\right)$ and $Y^{*}=\tau\left(\nu_{k-1}^{\prime \prime}, G_{k-2}\right)$. Lemmas 8, 10 and 11 show that

$$
\begin{aligned}
&\left|\tau\left(\nu_{k}^{\prime}, G_{k-1}\right)(\xi)\right|=\left|\bigcup_{j=0}^{\nu_{k}^{\prime}-1}\left\{X_{j}^{*}(\xi) \cup Y_{j}^{*}(\xi)\right\}\right| \\
& \leqslant \sum_{j=0}^{m-1}\left|Y_{j}^{*}(\xi)\right|+\sum_{j=\nu_{k}^{\prime}-m}^{\nu_{k}^{\prime}-1}\left|X_{j}^{*}(\xi)\right|+\sum_{j=0}^{\nu_{k}^{\prime}-m-1}\left|\left(X_{j}^{*} \cup Y_{j+m}^{*}\right)(\xi)\right| \\
&= \frac{m}{2 \nu_{k}^{\prime}}\left|Y^{*}(\xi)\right|+\frac{m}{2 \nu_{k}^{\prime}}\left|X^{*}(\xi)\right| \\
&+\frac{\nu_{k}^{\prime}-m}{\nu_{k}^{\prime}}\left|\left\{\left(\frac{1}{2} X^{*}+\frac{i}{2}\right) \cup\left(\frac{1}{2} Y^{*}+\frac{1-i}{2}+m\right)\right\}(\xi)\right| \\
& \leqslant \frac{m}{\nu_{k}^{\prime}}\left|G_{k-2}(\xi)\right|+\frac{\nu_{k}^{\prime}-m}{\nu_{k}^{\prime}}\left|H\left(\nu_{k-1}^{\prime}, \nu_{k-1}^{\prime \prime}, m, G_{k-2}\right)(\xi)\right| \\
& \leqslant \frac{m}{\nu_{k}^{\prime}}+\frac{\nu_{k}^{\prime}-m}{\nu_{k}^{\prime}}\left[\left\{1-\xi_{m}\left|G_{k-2}(\xi)\right|\right\}\left|G_{k-2}(\xi)\right|\right. \\
& \leqslant\left.+1-\xi_{m}\left|E_{n}(\xi)\right|\right\}\left|G_{k-2}(\xi)\right|+\operatorname{Const}\left[\frac{m+1}{\nu_{k-1}^{\prime}}+\left\{\prod_{j=0}^{k-2}\left(\nu_{j}^{\prime}+\nu_{j}^{\prime \prime}\right)\right\} \frac{\nu_{k-1}^{\prime}}{\nu_{k-1}^{\prime \prime}}\right] .
\end{aligned}
$$

These inequalities are valid with $\nu_{k}^{\prime}$ replaced by $\nu_{k}^{\prime \prime}$, and hence

$$
\begin{aligned}
\left|G_{k}(\xi)\right| \leqslant & \left\{1-\xi_{n}\left|E_{n}(\xi)\right|\right\}\left|G_{k-2}(\xi)\right| \\
& + \text { Const }\left[\frac{m+1}{\nu_{k-1}^{\prime}}+\left\{\prod_{j=0}^{k-2}\left(\nu_{j}^{\prime}+\nu_{j}^{\prime \prime}\right)\right\} \frac{\nu_{k-1}^{\prime}}{v_{k-1}^{\prime \prime}}\right] .
\end{aligned}
$$

Using this inequality for $k=n, n-2, \ldots, n-2(\iota(n / 2)+1)$, we have, with an absolute constant $C_{0}^{\prime}$,

$$
\begin{aligned}
\left|E_{n}(\xi)\right|= & \left|G_{n}(\xi)\right| \leqslant\left\{1-\xi_{m}\left|E_{n}(\xi)\right|\right\}^{\iota(n / 2)}\left|E_{n-2 \iota(n / 2)}(\xi)\right| \\
& + \text { Const } \sum_{l=0}^{\iota(n / 2)}\left[\frac{m+1}{\nu_{n-2 l-1}^{\prime}}+\left\{\prod_{j=0}^{n-2 l-2}\left(\nu_{j}^{\prime}+\nu_{j}^{\prime \prime}\right)\right\} \frac{v_{n-2 l-1}^{\prime}}{v_{n-2 l-1}^{\prime \prime}}\right] \\
\leqslant & \left\{1-\xi_{m}\left|E_{n}(\xi)\right|\right\}^{\iota(n / 2)}+C_{0}^{\prime} \rho_{n} .
\end{aligned}
$$


In the same manner as in the estimate of $\delta_{0}$, this yields that $\left|E_{n}(\xi)\right| \leqslant$ $\left(3 / n \xi_{m}^{\prime}\right) \log \left(10+n \xi_{m}^{\prime}\right)(m \leqslant \xi \leqslant m+1)$, where $\xi_{m}^{\prime}$ is defined by $1 / \xi_{m}^{\prime}=1 / \xi_{m}+$ $C_{0}^{\prime} n \rho_{n}$. Thus

$$
\begin{aligned}
\delta_{m} & \leqslant \frac{1}{m^{3}} \int_{m}^{m+1}\left|E_{n}(\xi)\right| d \xi \\
& \leqslant \frac{2}{m^{3} n}+\frac{1}{m^{3}} \int_{m+1 / n}^{m+1-1 / n} \frac{3}{n \xi_{m}^{\prime}} \log \left(10+n \xi_{m}^{\prime}\right) d \xi \\
& \leqslant \operatorname{Const}\left\{\frac{(\log n)^{2}}{n}+\rho_{n} \log n\right\} \frac{1}{m^{3}} \quad(1 \leqslant m \leqslant n) .
\end{aligned}
$$

By (6) and (7), we have

$$
\begin{aligned}
\sum_{m=0}^{n+1} \delta_{m} & \leqslant \sum_{m=0}^{n} \delta_{m}+\int_{n+1}^{\infty} \frac{d \xi}{\xi^{3}} \\
& \leqslant \operatorname{Const}\left\{\frac{(\log n)^{2}}{n}+\rho_{n} \log n\right\}\left\{1+\sum_{m=1}^{n} \frac{1}{m^{3}}\right\}+\frac{1}{2(n+1)} \\
& \leqslant \text { Const }\left\{\frac{(\log n)^{2}}{n}+\rho_{n} \log n\right\} .
\end{aligned}
$$

In the same manner, we have $\sum_{m=1}^{n+1} \delta_{-m} \leqslant \operatorname{Const}\left\{(\log n)^{2} / n+\rho_{n} \log n\right\}$. Consequently we have

$$
\operatorname{Bu}\left(E_{n}\right)=\sum_{m=-n-1}^{n+1} \delta_{m} \leqslant \operatorname{Const}\left\{\frac{(\log n)^{2}}{n}+\rho_{n} \log n\right\} .
$$

Now we put

$$
\begin{array}{ll}
\nu_{k}^{\prime}=n^{3} & (1 \leqslant k \leqslant n), \\
\nu_{k}^{\prime \prime}=n^{2}\left\{\prod_{j=0}^{\bar{k}-1}\left(\nu_{j}^{\prime}+\nu_{j}^{\prime \prime}\right)\right\} \nu_{k}^{\prime} & (1 \leqslant k \leqslant n),
\end{array}
$$

where $\nu_{0}^{\prime}=\nu_{0}^{\prime \prime}=1$. Then, by (5), we have $\rho_{n} \leqslant 2 / n$. By (8), we have $\operatorname{Bu}\left(E_{n}\right) \leqslant$ Const $(\log n)^{2} / n$. This completes the proof of (3). As easily seen, (2) and (3) yield the required equality in our theorem. This completes the proof of our theorem.

\section{REFERENCES}

1. A. M. Davie and B. $\Phi \mathrm{ksendal}$, Analytic capacity and differentiability properties of finely harmonic functions, Acta Math. 149 (1982), 127-152.

2. G. David, A lower estimate for the norm of the Cauchy integral operator on Lipschitz curves, preprint.

3. J. Garnett, Analvtic capacity and measure, Lecture Notes in Math., vol. 297, Springer-Verlag, 1972.

4. L. D. Ivanov, On sets of analytic capacity zero, Lecture Notes in Math., vol. 1043, Springer-Verlag, 1984 , pp. $498-501$. 
5. J. L. Journé, Calderon-Zygmund operators, pseudo-differential operators and the Cauchy integral of Calderon, Lecture Notes in Math., vol. 994, Springer-Verlag, 1983.

6. D. E. Marshall, Removable sets for bounded analytic functions, Lecture Notes in Math., vol. 1043, Springer-Verlag, 1984, pp. 485-490.

7. T. Murai, Boundedness of singular integral operators of Calderon type. VI, Nagoya Math. J. 102 (1986), 127-133.

8. L. A. Santaló, Introduction to integral geometry, Hermann, 1953.

Department of Mathematics, College of General Education, Nagoya University, Chikusa KU, NAGOYA, 464, JAPAN 